\title{
ASTROMETRY AND PHOTOMETRY OF 400 MILLION STARS BRIGHTER THAN 18 MAG
}

\author{
E. $\mathrm{H} \varnothing \mathrm{G}$ \\ University Observatory \\ $\emptyset$ stervoldgade 3 \\ 1350 Copenhagen K, Denmark
}

\begin{abstract}
A satellite mission for accurate astrometry and multi-colour photometry is discussed, similar in principle to the ESA Hipparcos mission and here called the Roemer mission. The limiting magnitude will be about $V=18 \mathrm{mag}$ while $13 \mathrm{mag}$ is the limit of the present Hipparcos mission. Luminosities of stars up to $2 \mathrm{kpc}$ away can be obtained, corresponding to a volume 10000 times larger than with Hipparcos. A mission of 5 years will provide an accuracy of 0.1 milli-arcsec at 12th magnitude for positions and parallaxes and 0.05 milli-arcsec for annual proper motions. This is achieved by a satellite using a mosaic of CCD detectors in the focal planes of two beam-combiner telescopes of $0.29 \mathrm{~m}$ aperture. The instrument is described and the expected performance with an input catalog of 400 million program stars is given.
\end{abstract}

\section{The Roemer mission}

The concept of an Hipparcos-like satellite for astrometry and photometry is presented giving five or six orders of magnitude improvement over the ESA Hipparcos mission, Perryman et al. (1992). This is obtained by CCD detectors in the focal plane of two beam combiner telescopes of $0.29 \mathrm{~m}$ aperture. It is practical to distinguish the two missions by the names Hipparcos and Roemer (after Ole Rømer (1644-1710), the discoverer of the finite speed of light, the inventor of new types of astrometric telescopes: the transit instrument and the meridian circle).

An accuracy of 0.1 milli-arcsec at $12 \mathrm{mag}$ is predicted for positions and parallaxes and 0.05 milli-arcsec for annual proper motion from a 5 year Roemer mission, compared with 2 milli-arcsec for parallaxes from Hipparcos at the same magnitude. The limiting magnitude will be about $V=18$ if an accuracy of 1 milli-arcsec is required, while stars fainter than $13 \mathrm{mag}$ cannot at all be observed by Hipparcos. The required input catalog of all program stars may comprise all 400 million stars brighter than $V=18$. It may be constructed from the one million stars brighter then $V=12$ in the Tycho catalog and the remaining stars obtained from measurement of photographic plates. After further scientific, technical and economic considerations an input catalog consisting of only a subset of the 400 million stars might be preferred. In the present context the full four hundred million stars program will be discussed. This corresponds to at least a factor 100000 improvement in statistical weight compared with Hipparcos when the accuracy and the number of stars are taken into account. Double star components will be separated down to 0.3 arcsec.

The resulting scientific impact can only be briefly described here. Parallaxes with a useful accuracy would be obtained for stars up to $2 \mathrm{kpc}$ away. The volume of space, ten thousand times larger than covered by Hipparcos, contains many objects of low space density: $\mathrm{O}$ and $\mathrm{B}$ stars, supergiants, intermediate population II stars, super metal rich stars, Cepheids, RR Lyr stars, open clusters etc. Determination of their distances will greatly 
improve our knowledge of stellar luminosities and of the distance scale of the Universe. The proper motions, and thus space velocities, of all these objects will be obtained with a high accuracy permitting studies of the dynamics of the Galaxy. The net of stars will constitute a position reference system of milli-arcsec accuracy, valid for many decades, and with a density about 2500 stars per square degree, sufficient for any CCD exposure at large telescopes. The high position accuracy is required for astrophysical purposes in order to locate optical objects well enough to match the resolution of better than 100 milli-arcsec which will be obtained in future, not only by the Hubble Space Telescope.

The photometric results will be equally interesting. A wide band photometry $W$ is obtained with an accuracy of $0.005 \mathrm{mag}$ at $V=12$ for each field crossing. Results from about 480 field crossings in 5 years will be available for studies of variability. All stars will obtain five-colour photometry (UBVRI) with an accuracy about 0.003 mag at $V=12$ and 0.06 mag at $V=18$ from a 5 year mission.

Solar system objects brighter than $V=18 \mathrm{mag}$, except Sun, Moon and Mercury, may be observed. Surface photometry of planets, planetary nebulae and galaxies may be obtained to a limit of about $20 \mathrm{mag} / \mathrm{arcsec}^{2}$. Astrometry of many quasars and cores of galaxies will be possible, important for relating to the extragalactic astrometric reference frame.

The CCD image detector is described in Section 2, the telescope and spacecraft in Section 3. Detailed performance figures for a Roemer mission given in Appendix A are based on the theoretical studies by Lindegren (1978) and Yoshizawa et al. (1985) and realistic assumptions on CCD performance etc.

\section{The CCD image detector}

The use of a CCD as detector in an astrometry satellite is of interest in view of the ten times higher quantum efficiency compared with the image dissector tube of Hipparcos, and because the light of many stars can be integrated simultaneously. Until recently the small size of available CCDs did not match the large field of one square degree and the high spatial resolution of an Hipparcos-like satellite. The large CCDs with millions of pixels now becoming available offer new possibilities, including sufficient dimensional stability and special purpose designs.

Two very different ideas for the use of a CCD should be discussed. The CCD image detector presented here places the CCD in the focus of the telescope and uses it as the accurate position measuring standard. The other option, discussed by $\mathrm{H} \varnothing \mathrm{g}$ and Lindegren (1992) for the Hipparcos-2 mission, is called a CCD modulation detector. It places a grid in the focus, as in Hipparcos, and images it onto a CCD, instead of the image dissector tube of Hipparcos. The CCD is then used only for intensity measurement and no high resolution or positional stability is required. High positional stability might be difficult to achieve because a cooling to $-70 \mathrm{deg} C$ necessarily introduces large temperature differences between the CCD and its connection to the telescope. But a dimensional stability inside a CCD of better than part-per-million, when first-order bulk motion and magnification changes are removed, has been reported by Buffington et al. (1990) for periods of 10 hours. Such stability makes a calibration of CCD irregularities feasible as required for the present purpose for which even better special purpose CCDs will be available in due time.

In the focal plane of a scanning satellite like Hipparcos all stars move in parallel 'along scan'. The direction and size of this 'stellar velocity' must be quite constant, and its actual value as function of time must be known within $10^{-4}$ which is feasible (van Leeuwen 1991, priv. comm.). A very accurate measurement shall only take place along the scan, not perpendicularly, the reason being that the beam combiner is an accurate measuring standard of large angles along scan only.

The focal plane measuring device may accordingly consist of long modulating slits per- 


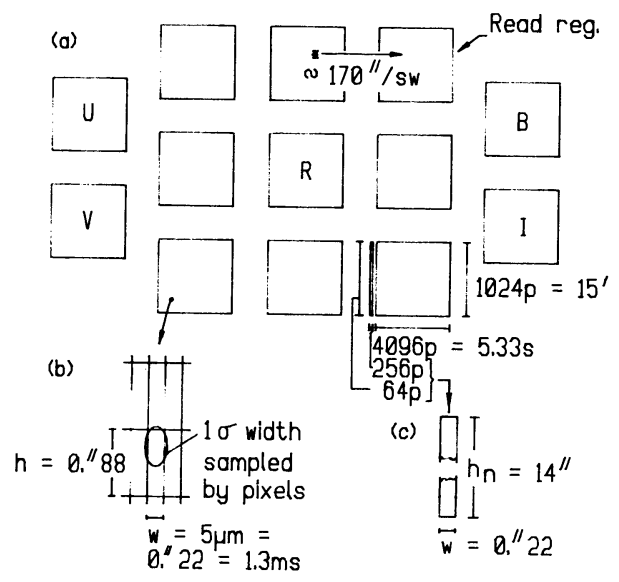

Figure 1. Focal plane arrangement of Roemer. (a) The stars drift across 13 chips, each containing a narrow and a wide CCD. Reading of the number of accumulated electrons (counts) takes place in a special register at the right edge of each CCD. Eight of the chips measure in a wide spectral band $W$ and five in the photometric bands $U B V R I$. (b) The $1 \sigma$ contour of the sampled diffraction image is shown superposed on the pixels of a wide CCD. (c) A pixel of a narrow CCD.

pendicular to the scan direction, as in Hipparcos, or of rectangular CCD pixels. A pixel width $w=0.22$ arcsec in direction of scan would match the optical resolution of a telescope with aperture $D=0.29 \mathrm{~m}$, cf. Section 4 . Since $5 \mu \mathrm{m}$ is the smallest width of pixel that can be manufactured a focal length of at least $f=4.7 \mathrm{~m}$ is required, implying a focal ratio of $0.29: 4.7=1: 16$.

The detector system in Figure 1a consists of 13 identical CCD chips, each containing a narrow and a wide CCD. The narrow CCD contains $256 \times 64$ pixels and the wide one $4096 \times 1024$ pixels of rectangular forms as shown in the Figure. A 'drift-scan' integration of the star image takes place while the image is moving or 'drifting' across a CCD. When it has moved the distance $w$ in $1.3 \mathrm{~ms}$ all charges of the CCD are quickly shifted by one pixel in direction of the image drift, so that the charge images are in fact only smeared by one pixel width.

Reading of the accumulated charges takes place in a reading register at the right edge of each CCD. Eight of the chips measure in a wide spectral band $W$ and five in the photometric bands $U B V R I$.

Sampling of the star image by a wide CCD is illustrated in Figure 2a. Single pixels may be read but usually the charges in four vertical pixels are added, giving one sample per $1.3 \mathrm{~ms}$ per star. Any star in the input catalog should be covered by about 16 samples, assuming rms errors of 0.5 arcsec for the positions in the input catalog and 0.2 arcsec for the real time attitude knowledge. Each of the samples corresponds to $5.3 \mathrm{~s}$ integration over the crossing of a wide CCD. The resulting 96000 samples/s (from $2 \times 13$ wide CCDs in two beam combiner telescopes and 400 million program stars) are transmitted to the ground, together with timing information etc.

The 'vertical' length of the pixels, i.e., perpendicular to the scan direction, is not important for the accurate measurement along scan. The value chosen here of $h=0.88$ arcsec for the wide CCDs will allow a measurement of the vertical position of reference stars with a precision about 0.1 arcsec if the four pixels in a vertical column are read for all bright reference stars. This precision is sufficient for the required attitude determination in real time and on ground, thus making starmapper slits as in Hipparcos superfluous. With say 

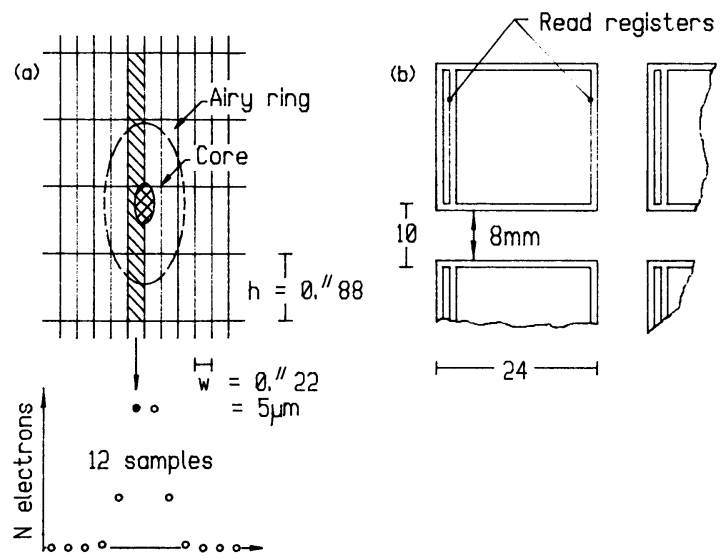

Figure 2. The sampling and the chips. (a) Sampling of the star image. Single pixels may be read but usually the number of electrons in four pixels (hatched) are summed into one sample (filled dot). Any star in the input catalog will be covered by about 16 samples and each of these corresponds to $5.3 \mathrm{~s}$ integration over the crossing of a wide CCD. (b) The chips, each containing a narrow and a wide CCD, are mounted on a frame with the required minimum edge-to-edge spacing.

one million reference stars available the required accuracy of 0.01 arcsec can be obtained in the subsequent data reduction on the ground.

The CCD chips (Figure $2 \mathrm{~b}$ ) are mounted on a frame of high thermal dimensional stability. Their surface shall follow the curved focal surface, taking the five thick glass colour filters into account. The wide CCDs in the $W$ band will permit linear accumulation of charge only for stars fainter than $V=13$. The narrow CCDs will be linear up to $V=7$ in the $W$ band, and up to $V=4$ or 5 behind a colour filter. These limits are expected because the linearity of present day pixels ends at 15000 electrons per $100 \mu \mathrm{m}^{2}$ and a star of $V=10$ will produce 95000 electrons/s, nearly half of these sometimes in one pixel. Saturation occurs at a three times higher charge, but future CCD technique is expected to permit ten times higher values.

\section{Telescopes and spacecraft}

The required focal length of $4.7 \mathrm{~m}$ derived in Section 2 is much longer than the $1.4 \mathrm{~m}$ of Hipparcos so that a very different optical system is needed. A field diameter of more than one degree is required in order to cover the sky with the same 'revolving' pattern as Hipparcos. A field of this diameter would give a large central obscuration in any on-axis telescope of focal ratio $1: 16$ and this would deteriorate the resolution for a given aperture. An off-axis system is therefore proposed.

Figure $3 \mathrm{~b}$ shows the two off-axis telescopes $\mathrm{A}$ and $\mathrm{B}$. The field at $F_{\mathrm{A}}$ is about 2 degrees off-axis and it is expected that a suitable Schmidt-like deformation can provide diffraction limited performance. The folding by two flat mirrors is for simplicity chosen so that all main axes lie in a single plane, but more compact designs may exist.

The angles between all stars along scan of Hipparcos are accurately determined due to the large and rigid basic angle between the two fields in combination with the relatively wide field of view. There is nevertheless a certain lack of rigidity (cf. Perryman and Hassan 1989, Section 3.6) which contributes a significant random error to all stars. This can be suppressed by the use of two beam combiner telescopes (Figure 3a) with different basic 
(a)

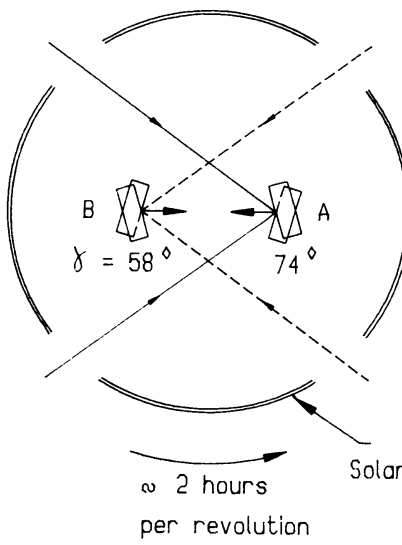

(b)

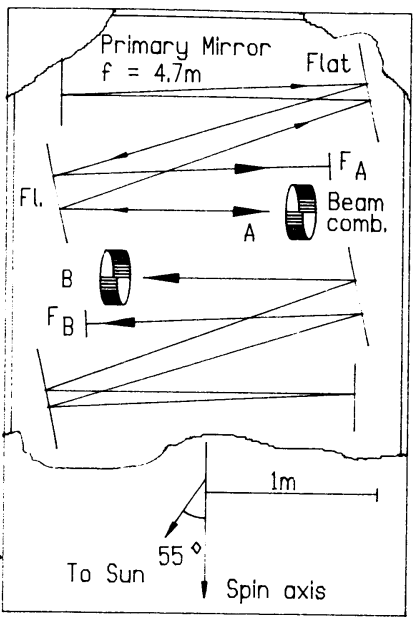

Figure 3. Optical system in case two telescopes are required, cf. the text. (a) Two beam combiner mirrors $\mathrm{A}$ and $\mathrm{B}$ with basic angles e.g. 58 and 74 degrees, shown in a projection perpendicular to the spin axis. (b) Two folded off-axis telescope systems with focal length about $5 \mathrm{~m}$ in a cylindrical spacecraft.

angles, e.g. 58 and 74 degrees, as discussed by Høg and Lindegren (1992). A study by Makarov (1991, priv. comm.) indicates that the effect would then become negligible in the Roemer project. It is, however, under investigation whether a single beam combiner telescope and a suitable data reduction would give sufficient rigidity so that the complication of having two telescopes can be avoided.

A very smooth rotation of the satellite is desirable for deriving the satellite attitude around all three axes more accurately than in Hipparcos. This would improve the resulting accuracy of star positions. The main torques disturbing a purely inertial rotation of Hipparcos at the geostationary distance are solar radiation pressure and reactions of the gyroscopes (used for measuring the attitude) generated by the satellite spin. The resulting attitude perturbations have to be compensated by firing of gas jets about every 10 minutes in Hipparcos in order to maintain the attitude within the required limits, whereas a purely inertial rotation would require much more seldom firings.

The interval between firings should be increased to at least 30 minutes, corresponding to 90 degrees of satellite rotation. This means that the perturbing torques just mentioned must be decreased to one tenth of their value for Hipparcos. Other torques from e.g. the gravity gradient and the magnetic field are still smaller. The solar radiation torque can be decreased by giving the satellite nearly rotational symmetry (Figure 3 ) and such form, surface and mass distribution that the resultant solar pressure vector goes nearly through the center of gravity. The gyro reaction can be sufficiently reduced by arranging the gyroscopes in pairs with anti-parallel spin.

The nominal revolving scanning law of Hipparcos should in principle be maintained. A study of this subject by Lindegren (1991, priv. comm.) has shown that revolving scanning is optimal, and superior to e.g. the Vlasov scanning scheme proposed by Chubey et al. (1990). An increase of the constant 'aspect angle' between spin axis and direction to the sun in the revolving scanning from the 43 degrees of Hipparcos to some 55 degrees would give a more uniform accuracy over the sky and more precise parallaxes. With this larger angle solar cells on a cylindrical spacecraft should provide sufficient power. Solar panels on hinges 
should in any case be avoided because thermal changes on the Hipparcos panels when the satellite entered the Earth's shadow have caused disturbing jumps of the attitude.

\section{Concluding remarks}

The data reduction for a Roemer mission will be much larger in pure quantity than the present Hipparcos/Tycho analysis, but it will in some ways be less complicated. All stars are observed for the same length of time in Roemer when they cross a CCD, instead of being observed with a complicated observing strategy; there is only one kind of detector and it observes the direct star image instead of modulated signals.

The Roemer payload is simpler than that of Hipparcos in some respects. All detectors are placed in the focal plane instead of having the voluminous arrangement of four photomultiplier tubes and two image dissector tubes, including redundant ones. There is no relay lens system which in Hipparcos contains 12 lenses giving considerable absorption and a geometric transmissivity of only 0.65 because it could not be made of sufficient aperture to transmit the entrance pupil, diffracted by the focal grid.

The hundred times larger number of photon detections per star provided by the new detectors compared with Hipparcos are basic for an improvement of precision. This can be translated into accuracy improvement in a new mission thanks to the lessons learnt from the Hipparcos mission.

Acknowledgements: It is a pleasure to thank R. Florentin Nielsen, J. Janesick, L. Lindegren and $\mathrm{H}$. Pedersen for information and discussions and I am grateful to T. Knudsen and L. Hansen for assistance with a computer program. This work was supported by the Danish Space Board.

\section{References}

Bessell M.S., 1990, PASP 102, 1181.

Buffington A., Hudson H.S., Booth C.H., 1990, PASP 102, 688.

Chubey M.S., Makarov V.V., Yershov V.N., Kanayev I.I., Fomin V.A., Streletsky Yu.S., Schumacher A.V., 1990, In: Lieske J.H. \& Abalakin V.K. (eds.) IAU Symp. No. 141, 77.

Høg E. and Lindegren L., 1992, IAU Symposium No. 156.

Lindegren L., 1978, In: Prochazka F.V. and Tucker R.H. (eds.) IAU Coll. No. 48, 197.

Makarov V., 1991, 'Periodical errors of the Hipparcos star abscissae', priv. comm., 6pp.

Perryman M.A.C. and Hassan H., 1989, The Hipparcos Mission, Volume I, The Hipparcos Satellite. ESA Publications Division, ESA-SP1111.

Perryman M.A.C., Høg E., Kovalevsky J., Lindegren L., Turon C., Bernacca P.L., Crézé M., Donati F., Grenon M., Grewing M., van Leeuwen F., van der Marel H., Murray C.A., Le Poole R.S., Schrijver H., 1992, A\&A, 258, 1.

Perryman M.A.C., Lindegren L., Murray C.A., Høg E., Kovalevsky J. et al., 1989, The Hipparcos Mission, Volume III, The Data Reductions. ESA Publications Division, ESASP1111.

Yoshizawa M., Andreasen G.K., Høg E., 1985, A\&A 147, 227. 


\section{APPENDIX A}

\section{Performance of a Roemer mission}

The following estimate of mean errors is based on photon statistical errors, since it is expected that the significant errors in Hipparcos from 'non-rigidity' and from parasitic stars can be neglected in Roemer. The measurement of a program star could be disturbed if another 'parasitic' star is situated within the $4 \times 4$ pixels $=3.1 \operatorname{arcsec}^{2}$ centered on the program star, cf. Figure $2 \mathrm{a}$. The probability of having a star brighter than $V=21$ within this area in either field-of-view is 0.024 for the mean sky and 0.10 if both fields are in the galactic plane. Therefore parasitic stars will give negligible disturbance in astrometry and photometry, compared with photon noise.

In the fundamental study of photoelectric astrometry by Lindegren (1978) formulae are given for the mean error of various image location estimators. The mean error of the optimum estimator for the diffraction image is

$$
\sigma_{\mathrm{X}}=1.000 \lambda /(\pi D \sqrt{N})
$$

where $D$ is the diameter of the circular telescope aperture, $\lambda$ is the (monochromatic) wavelength and $N$ the total number of photoelectrons in the image.

The modulating grid in Hipparcos gives a mean error 3.552 times larger. In other words, it has an astrometric efficiency of $E_{\text {grid }}=3.552^{-2}=7.9$ per cent. It must be concluded that the modulating grid, even when optimized for highest accuracy, makes bad use of the astrometric information contained in the diffraction image. This is due to the large fraction of light lost in the grid and to the bad matching of the grid to the diffraction pattern (cf. Lindegren 1978).

The CCD modulation detector discussed by $\mathrm{H} \varnothing \mathrm{g}$ and Lindegren (1992) makes use of the star light during a fraction $E_{\text {int }}=0.306$ of time. Its total astrometric efficiency is

$$
E_{\text {mod }}=0.306 \times E_{\text {grid }}=2.43 \text { per cent }
$$

relative to an optimum device, assuming that the basic photon detectors in both cases have the same quantum efficiencies.

The total astrometric efficiency, Eq.(7), of the proposed drift-scan detector shall now be derived. The astrometric mean error of a Gaussian image is given by Lindegren as

$$
\sigma_{\mathrm{x}}=1.000 \mathrm{~s} / \sqrt{N}
$$

where $s$ is the standard width of the image.

Let the diffractional star image, for simplicity, be approximated by a Gaussian of a standard width such that the position mean error estimated by an optimum method is equal to the position mean error of the diffraction image. It follows from (1) and (3) that this standard width is

$$
s_{\text {image }}=0.261(1.22 \lambda / D)
$$

where the bracket contains the radius of the Airy disk.

If the Gaussian image is superposed on a uniform background of intensity $k$ times the peak value of the Gaussian, the resulting $\sigma_{\mathrm{x}}$ may be calculated from Lindegren's exact formulae (11) and (28). The following simple formula is correct within 4 per cent for $k<1$

$$
\sigma_{\mathrm{X}}=(1.000 s / \sqrt{N}) \sqrt{1+4.77 k} /(1+0.2 k)
$$


Table 1. Predicted mean errors in astrometry and photometry for a 5 year Roemer mission. Columns 3 and 4 give errors for parallax and annual proper motions in milli-arcsec (mas), but asymptotic errors should be added as discussed in the text. Photometric errors are given for the $W$ band from $300-950 \mathrm{~nm}$, and for the five standard colours for stars of spectral type G0. Assumptions: Two beam combiner telescopes of $0.29 \mathrm{~m}$ aperture using 13 CCD chips in each focal plane.

\begin{tabular}{|c|c|c|c|c|c|c|c|c|}
\hline \multirow[b]{2}{*}{$\begin{array}{c}V \\
\mathrm{mag}\end{array}$} & \multicolumn{2}{|c|}{ Astrometry } & \multicolumn{4}{|c|}{ Photometry ( $W=$ Wide band $)$} & \multirow[b]{2}{*}{$\begin{array}{r}R \\
\mathrm{mag}\end{array}$} & \multirow[b]{2}{*}{$\begin{array}{r}I \\
\mathrm{mag}\end{array}$} \\
\hline & $\begin{array}{l}\text { par. } \\
\text { mas }\end{array}$ & $\begin{array}{l}\text { p.m. } \\
\text { mas }\end{array}$ & $\begin{array}{c}W \\
\mathrm{mag}\end{array}$ & $\begin{array}{c}U \\
\mathrm{mag}\end{array}$ & $\begin{array}{r}B \\
\mathrm{mag}\end{array}$ & $\begin{array}{r}\hat{V} \\
\mathrm{mag}\end{array}$ & & \\
\hline 10 & 0.02 & 0.01 & 0.000 & 0.003 & 0.001 & 0.001 & 0.001 & 0.001 \\
\hline 11 & 0.03 & 0.01 & 0.000 & 0.005 & 0.002 & 0.001 & 0.001 & 0.001 \\
\hline 12 & 0.05 & 0.02 & 0.000 & 0.008 & 0.003 & 0.002 & 0.002 & 0.002 \\
\hline 13 & 0.07 & 0.04 & 0.001 & 0.013 & 0.004 & 0.004 & 0.004 & 0.004 \\
\hline 14 & 0.12 & 0.06 & 0.001 & 0.021 & 0.007 & 0.006 & 0.006 & 0.006 \\
\hline 15 & 0.19 & 0.10 & 0.002 & 0.036 & 0.011 & 0.010 & 0.009 & 0.010 \\
\hline 16 & 0.31 & 0.16 & 0.003 & 0.067 & 0.018 & 0.016 & 0.015 & 0.016 \\
\hline 17 & 0.54 & 0.27 & 0.004 & 0.135 & 0.032 & 0.029 & 0.027 & 0.028 \\
\hline 18 & 0.99 & 0.50 & 0.009 & 0.302 & 0.064 & 0.056 & 0.052 & 0.055 \\
\hline 19 & 1.96 & 0.98 & 0.018 & - & 0.137 & 0.121 & 0.112 & 0.119 \\
\hline
\end{tabular}

In the CCD image detector of Figure 1a 'drift-scan' integration of the star image takes place while it is drifting across the CCD. When it has moved the distance $w$ all charges of the CCD are quickly shifted by one pixel in direction of the image drift, so that the images are in fact only smeared by one pixel width.

This is equivalent to the image being folded with two uniform functions of width $w$, one due to the pixel sampling and one due to the drift during sampling. Each of these will add quadratically a standard width of $w / \sqrt{12}$ to $s_{\text {image }}$ resulting in a total sampled standard width given by

$$
s_{\text {tot }}^{2}=s_{\text {image }}^{2}+w^{2} / 12+w^{2} / 12
$$

The total astrometric efficiency is therefore according to (3)

$$
E_{\text {drift-scan }}=s_{\text {image }}^{2} / s_{\text {tot }}^{2}=1 /\left(1+\left(w / s_{\text {image }}\right)^{2} / 6\right)
$$

For example, for $D=0.29 \mathrm{~m}$ and $\lambda=600 \mathrm{~nm}$ we obtain $s_{\text {image }}=0.136$ arcsec. The resulting efficiency of drift-scan is 90 and 70 per cent for $w=0.111$ and 0.22 arcsec, respectively. This assumes that background and all other error sources than Poissonian photon noise are neglected in both cases. Even the 70 per cent efficiency is 30 times higher than the efficiency of the CCD modulation detector.

Equation (5) applied to the image detector does not include the loss of precision due to discrete sampling. It is expected from Yoshizawa et al. (1985) that this loss would be only about 5 per cent using a maximum-likelihood estimation, in spite of the considerable undersampling of the image.

The mean error of an observed magnitude is obtained from

$$
\sigma_{\mathrm{m}} \equiv 2.5 \log \mathrm{e} \sigma(\mathrm{N}) / \mathrm{N}=1.086 \sqrt{\mathrm{N}_{\mathrm{star}}+10 \mathrm{~b}} / \mathrm{N}_{\text {star }}
$$


where $N$ is the observed counts (i.e., the number of generated electron charges) from star plus background, $N_{\text {star }}$ is the counts due to the star and $b$ counts/sample is the background. Eq.(8) assumes that $N$ is the sum of 5 samples centered on the star and that $b$ is obtained from 5 samples besides of the star, cf. Figure 2a. This is a conservative estimate compared with that from a maximum-likelihood estimation.

The mean errors given in Section 1 for a 5 year Roemer mission are derived from Table 1. This table is based on the following assumptions: Stars of visual magnitude $V$ and spectral type $G 0$; a background of $b=30$ counts per sample as expected from a read noise of 2 electrons, plus sky and straylight as in the Hipparcos requirements, corresponding in total to 4 stars of $V=21.8$ per $\operatorname{arcsec}^{2}$ of $B-V=0.7$; two beam combiners of aperture $D=0.29 \mathrm{~m}$; mirror reflectivities from Perryman and Hassan (1989) Table 5.2; colour filter passbands according to Bessell (1990); quantum efficiencies of the thinned back-illuminated CCDs of $Q E=0.56,0.80,0.65,0.42$ at $300,650,800,900 \mathrm{~nm}$, respectively; a scan speed $v=168.75 \mathrm{arcsec} / \mathrm{s}$ giving $18.1 \mathrm{crossings} / \mathrm{star} /$ year/CCD; a fractional dead time of 0.10 due to Earth occultations. Furthermore, a factor of 1.5 has been applied to all photon noise errors, both for astrometry and photometry. This is the factor found empirically for Hipparcos astrometry in connection with Table 2 of $\mathrm{H} ø \mathrm{~g}$ and Lindegren (1992) and in fact confirmed by the Hipparcos observations. The performance figures given in Table 1 are therefore supposed to be quite realistic or even conservative.

The detection rates for a star of $V=10$ were calculated. For Hipparcos the average rate behind the grid was found to be $787 \mathrm{~Hz}$, in reasonable accordance with the $600 \mathrm{~Hz}$ predicted before launch in Perryman et al. (1989, [12.3]), and with the observed rate. The equivalent detection rate for Roemer calculated by the model is $95000 \mathrm{~Hz}$ in the $W$ band at $V=10.0$.

The mean errors given in Table 1 will be too optimistic for the bright stars. An asymptotic mean error must be added, especially to the astrometric errors. Supposedly conservative asymptotic errors of 0.1 milli-arcsec for parallaxes and positions and 0.05 milli-arcsec for annual proper motions have been assumed in obtaining the mean errors given in Section 1 for a 5 year mission. 